\title{
ON THE LUNAR SECULAR ACCELERATION: A POSSIBLE APPROACH *
}

\author{
V. Protitch-Benishek, M. B. Protitch \\ Astronomical Observatory \\ Volgina 7 \\ Yu-11050, Belgrade \\ Yugoslavia
}

ABSTRACT. The secular quadratic term in the expression of the Moon's longitude has been introduced empirically after the conclusion that its mean motion is not constant (Halley, 1695).

But, the explanation of this term and also of its numerical evaluation presented and still presents in our time great difficulties. All efforts, namely, to obtain an exact agreement between observed and theoretical value of Moon's secular acceleration were unsuccessful: the first of these two values exceeds always the second one by a very large amount. This discordance and unexplained residuals $(O-C)$ in the mean longitude of the Moon gave rise finally to the statement that these are due to a retardation and irregularity in the Earth's rotation. But, after hardly a fifty years, this hypothesis revealed even more new difficulties and questions concerning also the problem of stability of the Earth-Moon system. It seems that there is a true reason for which this problem occurs as one of the unsolved problems of Celestial Mechanics (Brumberg and Kovalevsky, 1986; Seidelmann, 1986).

However, such an inconvenience can be, nevertheless, avoided if the conditioned periodical functions are applied as theoretical basis to find the solution of the perturbed motion of celestial bodies relatively to their central mass. Without entering here into details, only recalling that in this case the analytical expression for the longitude is defined by:

$$
L=L_{o}+n t+\sum A_{i} \sin \left(\alpha_{i} t+\beta_{i}\right), \quad(i=1,2, \ldots, n)
$$

i. e. without any other $t$-term except the linear one.

The analogous relations are deduced for the elements $\bar{\omega}, \Omega$, longitude of pericenter and node, respectively. The orbital elements $a, e, i$, semi-major axis, eccentricity and inclination, appear as cosine functions, while $t$-terms do not exist in an explicite form. Generally, the unknowns $L_{o}, n, A, \alpha, \beta$, are to be found from the observations.

If we assume these solutions as correct and very well adapted to describe also the motion of the Moon, the unexplained residuals in its longitude can be expressed as:

$$
\delta L=\delta L_{o}+\delta n t+\sum C_{i} \sin \left(\alpha_{i} t+\beta_{i}\right)
$$

* This paper in extenso will be published in the Bull. Obs. Astron. Belgrade, 142, 1990. 
where the sum of periodical terms represents the inequalities not involved in actual theory of lunar motion (Main problem, omitting secular acceleration as a whole).

In view of the above comments, we shall now summarise the main result found on the basis of residuals $(O-C)$ relatively to Brown's tables of the Moon. The reduction of the residuals $(O-C)_{B r o w n}$ to the quasi-keplerian values $\delta L$ was performed applying the corrections for a secular quadratic term, the great empirical term and the great Venus term $(A)$, introduced by Hansen to remove the large excesses in Airy's series of Greenwich meridian observations of the Moon during 1750-1830. Then, we find:

$$
\delta L=(O-C)_{B r}+7.14^{\prime \prime} T^{2}+10.71^{\prime \prime} \sin \left(140.0^{\circ} T+240.7^{\circ}\right)+A,
$$

( $T$ counted in Julian centuries from 1900.0), or:

$$
\delta L=B+G+A \text {, }
$$

if the values of the fluctuation $B$ and corresponding corrective term :

$$
G=+4.65^{\prime \prime}+12.96^{\prime \prime} T+12.36^{\prime \prime} T^{2}
$$

are introduced instead of forgoing numerical part in expression for $\delta L$.

The data we have utilised were: (1) the values $B$, adopted from Brouwer's paper (1952), for a period 1685-1950, and (2) the same values, but derived on the basis of the corrections $\Delta T=E T-U T$ for the following thirty five years. After removal of the long-periodical inequality, which satisfies entirely all Ptolemaic, Arabian and medieval lunar eclipses, the reminder part of residuals $\delta L$ was analysed. The mentioned inequality of a period of 64686 lunations or, 5230 years, comprises also the corrections $\delta L_{o}$ and $\delta n$. Therefore:

$$
\Delta(\delta L)=\delta L-\delta L_{1}
$$

Determining the parameters of periodical terms, the sixteen smoothed and equally displaced values of residuals are chosen. Kühnen's method and the least square method were applied in two steps: firstly, with a four periods and then, with three additionals, derived from the remain residuals. It is, however, important to notice that the computed periods are very close to the times needed for restoring the identical conjunction (or opposition) of the lunar ascending node and also of the perigee with the Sun at the same point of the ecliptic. During the determination of the amplitudes $C$ these last intervals of time were accepted as true.

Reversing the process, the fluctuations $B_{C}$, corresponding to a five-years intervals and also to all of the utilised data were calculated. The computed values are in a good agreement with the observed ones. This fact enables us to expect that here presented approach can contribute not only to solve the problem of discordances between observations and theory, but also to find the true answer to many important questions of Celestial Mechanics.

\section{REFERENCES}

Halley,E. : 1695, Phyl. Trans. Roy. Soc. London, 218.

Brumberg,V. A. ,Kovalevsky,J. : 1986, Celest. Mech. , 39, 133-140.

Seidelmann,P. K. : 1986, Celest. Mech. , 39, 141-146. 